Salaka Sanou

Salaka Sanou est Maître de Conférences (HDR) au Département de Lettres Modernes, à l'Unité de Formation et de Recherches en Lettres, Arts et Communication (UFR/LAC), Université de Ouagadougou.

\section{Littérature et masque : une étude comparée de leur fonctionnement comme institutions}

\title{
Littérature et masque : une étude comparée de leur fonctionnement comme institutions
}

Le fonctionnement de la littérature comme une institution est connu et a été suffisamment développé dans les études littéraires ; cela a permis aux différents théoriciens de définir l'institution et de montrer l'autonomie du champ littéraire producteur de valeurs qui sont transmises selon des codes. Le masque en Afrique est une réalité culturelle et cultuelle au-delà de sa dimension artistique ; il constitue, dans les sociétés où il existe, une référence sociale, un régulateur de la vie sociale. En particulier chez les Bobo du Burkina Faso, il est l'élément, la structure de socialisation par excellence de l'individu. En observant le fonctionnement de la littérature et du masque, nous avons été frappé par des similitudes qui nous ont conduit à nous demander si l'on ne pouvait pas considérer le masque comme une institution au même titre que la littérature. Pour répondre à notre interrogation, nous avons procédé à une approche théorique des deux phénomènes pour ensuite mettre en parallèle leur fonctionnement ; et notre conclusion est formelle : le masque est une institution comme la littérature et à ce titre, il peut faire l'objet d'une étude sous cet angle. Mots-clés : littérature, sociologie de la littérature, masque, institution.

\section{Introduction}

L'intitulé de notre étude peut paraître curieux ou inintéressant selon le niveau de connaissance que l'on a du masque en particulier car la conviction est établie pour les études littéraires que la littérature fonctionne comme une institution. Mais alors quel rapprochement avec le masque?

La curiosité que peut susciter notre étude réside dans la manière de considérer le masque : en effet, si celui-ci est pris comme un simple objet d'art destiné à des décorations de salons ou à des expositions en musée, il y a évidemment à se demander comment le masque peut être analysé comme une institution. Passé le temps de la curiosité, on peut aussi se poser des questions sur l'intérêt que peut avoir une telle étude car, pour ceux qui connaissent le masque comme un être vivant dont la vie est réglementée et liée à des pratiques rituelles bien établies, il y a évidence que le masque est une institution ; de ce point de vue notre étude ne viendrait qu'enfoncer une porte déjà grandement ouverte.

Entre les deux positions nous avons décidé de jeter un pont pour, d'une part, apporter l'éclairage aux curieux et, d'autre part, synthétiser des connaissances pro- 
fanes et éparses pour permettre d'avoir des éléments pertinents non seulement basés sur une observation attentive mais aussi sous-tendus par une théorie éprouvée. Ainsi, nous intéressant à la sociologie de la littérature, nous avons su saisir et analyser le fonctionnement de la production littéraire, notamment au Burkina Faso, comme une institution. Ces recherches nous ont permis d'identifier localement les différents acteurs et leurs rôles respectifs dans le procès de la littérature (Sanou 2003).

Mais, à côté de cette préoccupation, nous nous sommes aussi intéressé aux masques dans un premier temps par sentimentalisme (étant nous-même issu d'une société de masque) et par la suite comme un véritable objet de recherche à travers la mise en place d'un groupe de recherche qu' est le Groupe de recherche en Esthétique littéraire et artistique négro-africaine à l'Université de Ouagadougou.

$C^{\prime}$ est ainsi que nous avons constaté des similitudes entre le fonctionnement de l'institution littéraire et celui du masque : au fur et à mesure de la progression dans nos investigations, il s'est imposé à nous la nécessité de systématiser ces constats, c'està- dire de les ordonner, les organiser afin d'en tirer des conclusions qui peuvent servir de pistes à approfondir. C'est la raison d'être de cet article qui, en tant qu'innovation théorique, peut surprendre par son contenu mais qui, tout en n'apportant pas de nouveaux éclairages sur la nature réelle du masque dans le contexte africain, ne présente pas moins le masque comme objet de recherche sous un éclairage nouveau. Pour ce faire, il nous est paru nécessaire de rappeler les caractéristiques et le fonctionnement de l'institution littéraire à partir des analyses de Jacques Dubois. Ensuite, pour une bonne compréhension de la démarche, il nous faut expliquer le fonctionnement et l'importance du masque chez les Bobo du Burkina Faso (qui, à quelques variances près, peuvent être généralisés à l'ensemble du continent). Enfin nous procéderons à une «mise en commun » des caractéristiques de la littérature et du masque pour conclure à l'existence d'une institution du masque à l'image de l'institution littéraire.

\section{L'institution littéraire}

Dans son acception générale, l'institution c'est la reconnaissance d'un sous- système social par l'Etat, c'est sa légitimité consacrée par l'appareil étatique pour en faire un maillon du fonctionnement et de la présentation de la société. Cependant, le mot institution peut couvrir sur le plan sémantique quatre domaines que nous pouvons schématiser comme suit :

- l'institution c'est l'action d'instituer quelque chose c'est-à-dire de le rendre pérenne en élaborant son mode de fonctionnement à travers une codification. Dans ce sens, elle renvoie à «établissement » ; « fondation, » ; « instauration » ;

- l'institution c'est aussi la chose instituée ; c'est le cas par exemple des organisations internationales comme l'organisation des Nations unies, l'Union afri- 
caine qu'on peut désigner sous le vocable d'institution

- l'institution c'est encore ce qui est établi par les hommes, le modèle réduit d'un système social dont la légitimité est consacrée par l'Etat : c'est par exemple le cas de la famille, l'école, le mariage, l'armée, etc. La caractéristique principale dans ce cas c'est la fonctionnalité dans le système social, c'est-à-dire le rôle qu'elle joue mais aussi son mode de fonctionnement qui lui permet de jouer ce rôle ;

- enfin le mot institution peut avoir aussi un sens collectif et didactique ; dans ce cas l'institution c'est « l'ensemble des structures organisées tendant à se perpétuer dans chaque secteur de l'activité sociale »(Grassin), ce qui nécessite l'observance d'une distinction entre ces différentes activités sociales : c'est ainsi qu'on parlera d'une institution juridique, littéraire, artistique, etc. L'institution dans ce sens renvoie à la notion de système comme la structure d'une totalité qui est composée d'un ensemble de sous-systèmes dont chacun est la reproduction, à une échelle réduite, du système.

A partir de ces différentes acceptions du mot institution, comment en est-on arrivé à parler d'institution littéraire, d'institution de la littérature ? Sans vouloir refaire ici l'histoire de ce concept relativement récent, il faut dire que c'est l'Américain Harry Levin qui a introduit en 1945 - 1946, dans un article intitulé Literature as an institution dans la revue Accent (Vol 6) ce concept de la vie littéraire. Dans son entendement, l'institution est inséparable du concept de système ; parler d'institution littéraire revient à considérer la littérature comme une pratique autonome, un champ, pour reprendre le mot de Pierre Bourdieu, entretenant des liens avec les éléments sociaux organisés et structurés par un type de société.

Le mérite revient à Jacques Dubois d'avoir systématisé l'analyse de la vie littéraire en terme d'institution à travers son ouvrage L'institution de la littérature (1986). Dans la constitution du cheminement de la réflexion sur une « conception institutionnelle de la littérature moderne ", il retient trois auteurs dont les œuvres théoriques vont jeter les jalons : Jean-Paul Sartre (Qu'est-ce que la littérature? Situations II) Roland Barthes (Le degré zéro de l'écriture) et Pierre Bourdieu avec ses travaux sur le champ culturel. «En rupture par rapport aux philosophies idéalistes de l'art, ces trois auteurs saisissent le littéraire comme produit d'une société historique et l'appréhendent à l'intérieur des rapports de classe » (Dubois 1986: 9). Il s'agit d'une rupture tridimensionnelle dans le procès de la littérature : rupture dans la perception de la fonction de l'écrivain, de l'écriture et de la littérature comme bien symbolique.

En considération de cette approche, trois instances peuvent être retenues comme constituant des bases de l'institution littéraire :

- L'écrivain en tant que créateur, point de départ de la vie littéraire. Il constitue l'instance de création avec ses systèmes de codes esthétiques et de régimes 
rhétoriques dont la connaissance permet de déterminer le rôle dans la vie littéraire. En effet, l'écrivain a un itinéraire et des habitus dans le champ littéraire qui permettent de dégager «les indices de la légitimité et de la reconnaissance acquise [...], définir le degré de lisibilité et d'autorité détenu par chacun » (Durand 2001: 34).

- L'édition : les créations des écrivains resteraient de simples manuscrits dans leurs tiroirs et seraient inconnues et inaccessibles au public si l'éditeur n'intervenait pas comme l'instance de médiation entre le créateur et ses lecteurs. L'éditeur en tant que maillon essentiel de la vie littéraire influence celle-ci à travers sa ligne éditoriale qui détermine les choix des manuscrits et les créneaux de ses interventions. C'est l'instance de production et de distribution du livre qui met en place un système de tri, d'analyse, d'appréciation des manuscrits à partir duquel il s'engage dans la production c'est-à-dire la matérialisation $d u$ manuscrit du livre et la distribution ou la mise en livre dans le circuit de la « consommation ». L'édition est une activité à cheval entre le culturel et le commercial et c'est cette situation d'ambivalence qui justifie et explique le mode d'organisation et de fonctionnement dont elle se dote en fonction de sa capacité. Nous n'allons pas ici décrire ni analyser cette organisation et ce fonctionnement.

- La lecture ou réception de l'œuvre littéraire. Des spécialistes comme Jean-Paul Sartre ou Robert Escarpit ont montré que le destin final du livre est la lecture qui est multidimensionnelle, ce qui a donné naissance à la théorie de la réception littéraire. Par réception littéraire il faut entendre les codes (esthétiques, sociaux, culturels, philosophiques, etc.) qui permettent aux lecteurs d'accéder à l'œuvre, à sa substance et ses sens. Comme on peut l'imaginer, la multitude de ces codes entraîne aussi une multitude de lectures, d'approches du texte littéraire. C'est ce qui explique aussi la multitude des instances de légitimation c'est-à-dire « un rouage institutionnel remplissant une fonction dans l'élaboration, la définition et la légitimation d'une œuvre »(Dubois 1986 82). Ainsi, légitimer une œuvre littéraire c' est lui reconnaître une conformité à un certain nombre de critères préexistants, clairement définis ou instinctivement reconnus par l'instance concernée. Parmi les principales instances de légitimation, on peut citer le salon ou la revue littéraire, la critique, l'académie, l'école, chacune remplissant une fonction précise dans la vie de l'œuvre littéraire. Selon Jacques Dubois, « $1^{\circ}$ le salon ou la revue supportent $l^{\prime}$ émergence $; 2^{\circ}$ la critique apporte la reconnaissance ; $3^{\circ} 1^{\prime}$ académie engage, par ses prix ou ses cooptations, la consécration ; $4^{\circ} l^{\prime}$ école, avec ses programmes et ses manuels, intègre définitivement à l'institution et garantit la conservation »(Dubois 1986: 87).

L'institution de la littérature apparaît alors comme l'étude du fonctionnement de la littérature sur la base d'une organisation dans laquelle chaque acteur joue un rôle qui 
s'est imposé dans la vie de l'œuvre littéraire. Cette organisation a été suffisamment mise en lumière par la sociologie de la littérature c'est-à-dire une étude de la littérature historiquement constituée en objet social relativement autonome. Elle part de l'écrivain conçu comme une trajectoire individuelle en passant par tout le processus de production de l'œuvre littéraire pour terminer par prendre en compte la lecture comme point final de la création littéraire. La sociologie de la littérature a donné une dimension nouvelle aux études littéraires en considérant la littérature comme une pratique autonome, « un champ dont les liens avec les éléments sociaux sont organisés et structurés par un certain type de société» (Grassin).

\section{Le masque africain}

Le mot masque est un terme ambigu dont l'utilisation que nous faisons ici est loin de sa signification courante en arts du spectacle par exemple. Le masque comme réalité socioculturelle en Afrique est loin de correspondre à son acception occidentale. Mieux, le mot français traduit mal cette réalité car il a une dimension réductrice qui se limite généralement à considérer le masque comme un déguisement. Afin de mieux présenter le masque comme phénomène socioculturel en Afrique, nous allons en donner les définitions issues des usages qui en sont faits.

Le Grand Larousse de la Langue française donne plusieurs définitions du masque : au niveau du corps humain, il peut s'agir d'un faux visage, de l'aspect réel d'un visage humain ; au niveau des arts plastiques, le masque peut être la reproduction d'un visage humain ou animal ; le masque peut aussi être un simple objet à l'image des masques à gaz, d'une élévation de terre, d'un abri en acier, etc. Il existe un autre mot dont l'étymologie se rapporte au masque : c'est la mascarade qui peut aussi être définie de plusieurs manières ; il peut s'agir d'un divertissement, d'une réunion ou défilé de personnes déguisées, d'un accoutrement ou déguisement ridicule ou alors d'une attitude hypocrite.

Le masque peut paraître donc comme un déguisement ou une dissimulation qui a donc un triple aspect :

- le camouflage c' est-à-dire une disparition, une perte factice de d'individualité, d'où la dissimulation ;

- le travesti c'est-à-dire l'imitation ou l'adoption d'une apparence définie ou trompeuse ; $c^{\prime}$ est la métamorphose chezl'homme ;

- l'intimidation qui est un procédé tendant à déclencher chez le spectateur un effroi hyperbolique.

Comme on peut le constater, toutes ces définitions et ces classifications montrent bien la complexité du concept et de sa compréhension. Nous allons y puiser pour mieux cerner le phénomène du masque en Afrique en partant de son étude au Burkina Faso, 
dans un groupe ethnique où il occupe une place centrale dans l'organisation et le fonctionnement de la société : les Bobo.

Les sociétés africaines traditionnelles, originellement et fondamentalement animistes, ont toujours établi une hiérarchie dans leurs relations à Dieu, être suprême et créateur de l'univers et de tout ce qui le peuple. Pour accéder à cet être suprême l'homme doit passer par une série d'intermédiaires dont l'importance respective est fonction de la place par rapport à Dieu. C'est ainsi par exemple que chez les Bobo du Burkina Faso, on a schématiquement et par ordre décroissant : Dieu (Wuro), les Ancêtres (Sompulala), la Brousse (Sogo), le masque (incarnation de Do), le village Kiri, le site d'habitation de l'homme), et les autres puissances spirituelles improprement appelées «fétiches» (collectives et individuelles).

Ainsi qu' on le voit, le masque occupe dans cette société une place importante liée au rôle qu'il joue dans l'organisation et le fonctionnement de la société. Dans cette société, le masque relève d'abord du spirituel : il est l'incarnation de la quatrième puissance spirituelle qu' est $D o$. On peut comprendre facilement que cette incarnation soit liée à un mythe dans lequel les autres puissances supérieures à Do auront un rôle prépondérant. Des multiples versions de ce mythe, nous retenons que le masque s'est révélé à l'Homme (tantôt l'homme, tantôt la femme selon les versions) dans la Brousse comme un être bizarre, étrange, en tout cas inhabituel et inconnu de lui. Face à la frayeur provoquée par sa vue chez l'homme, il va le rassurer en lui expliquant la démarche et les procédés à suivre pour qu'il lui soit utile. C'est ainsi que le rituel de la sortie des masques (toujours venant de la brousse) sera institutionnalisé et le masque considéré comme un être sacré de la brousse. Pour l'humaniser, il relèvera d'une puissance, $D o$, dont le siège sera au village, avec les hommes.

De ce qui précède, on peut retenir que l'origine du masque est liée au surnaturel, en rapport avec ce que d'aucuns ont appelé divinités mais que nous nommons puissances spirituelles du fait du caractère monothéiste de cette société bobo. ${ }^{1} \mathrm{Il}$ a donc une origine divine parce qu'émanation directe de la volonté divine. C'est la raison qui explique qu'il suscite crainte et respect de la part des hommes devant ce qui leur paraît relever d'une force mystérieuse et surnaturelle.

En tant qu' expression symbolique de certains aspects du surnaturel, le masque est toujours identifié (ou tend à s'identifier) à l'être qu'il représente. On comprend dès lors que les sociétés africaines traditionnelles ne distinguent pas, dans le masque, le porteur, c'est-à-dire l'être humain, du maquillage c' est-à-dire l'habillement dont il se couvre. Il y a comme une communion entre l'être humain (porteur du masque) et l'être surnaturel qu'il représente : $c^{\prime}$ est le principe de l'incarnation qui fait du masque en Afrique une puissance spirituelle dotée de pouvoirs en fonction de ce qu'il incarne aux yeux de la communauté. C'est ainsi que chez les Gurunsi, une ethnie du Burkina Faso, les différents masques zoomorphes (buffle, phacochère, lièvre, hyène, crocodile, lion, etc.) adoptent sur la scène, au moment de leurs prestations (ou hors 
prestations) les comportements, les attitudes et les actes couramment reconnus à l'animal en question. Donc, au-delà de la figuration sculptée de l'animal, il y a sa reconnaissance à travers ce qu'il fait.

Dans ces conditions il y a une influence de jeu de «l'acteur », c'est-à-dire le porteur du masque, sur le public qui accepte volontiers cette métamorphose des rôles. Cela a comme conséquence sa métamorphose à lui car, lors de la prestation des masques, il se retrouve dans une ambiance tout à fait différente de son ambiance habituelle : c'est qu'il se trouve comme transporté dans ce monde de l'au-delà, du surnaturel dont relève le masque.

Après cette présentation des différentes acceptions du masque, nous allons analyser la nature et la fonction du masque africain en les illustrant à partir du masque chez les Bobo, un groupe ethnique de l'ouest du Burkina Faso. Peuple fondamentalement animiste, les Bobo accordent une place primordiale au masque dans la vie et l'organisation de leur société. C'est ainsi que le masque se trouve au centre de toutes les étapes importantes de la vie de l'individu. C'est la raison pour laquelle nous allons partir de la vie du masque chez les Bobo pour analyser son fonctionnement, ce qui va nous permettre de mettre ce fonctionnement en parallèle avec celui de la littérature et de conclure que le masque et la littérature fonctionnent tous les deux comme une institution.

\section{Nature du masque}

Que représente donc le masque chez les Bobo ? Après observation et analyse, on peut affirmer que le masque bobo a trois dimensions :

\section{Le masque est un être sacré}

Parler du masque comme un être et non un objet peut surprendre plus d'un ; mais en réalité c'est sa nature même qui amène à le considérer comme un être : en effet, le masque est lié à un culte religieux, d'aucuns même affirment que c'est la religion dans les sociétés où il en existe. Son culte est lié à Do chez les Bobo, la quatrième référence spirituelle comme nous l'avons vu précédemment. En tant que culte, il est fondé sur trois piliers que sont la bouche, le secret et le mystère : la bouche doit savoir taire le secret du masque afin de sauvegarder son mystère. Cela est en rapport avec son mythe de révélation à l'homme qui, pour pouvoir le ramener de la brousse au village, s'est engagé à reproduire ce mythe à chaque sortie de masque. C'est ainsi que chaque fois que le masque doit participer à un rituel, il faut qu'il vienne de la brousse, que l'on recrée ce processus par lequel l'homme l'a « dompté » en l'amenant au village. Et pour rappeler cette origine le masque est appelé $k a n$ fra $(k a n=$ brousse, fra = être, objet) c' est-à-dire « être de la brousse » dans la langue bobo. Pour montrer qu'il s'agit d'un être, d'un objet animé, il est aussi appelé son yeru (son = homme, personne, yeru = âme) ou «âme de l'homme». 
$C^{\prime}$ est en considération de cette origine et de cette nature du masque qu'il est présent aux événements essentiels de la vie de l'homme : naissance, mort (funérailles), réjouissances, etc., événements socioculturels d'importance pour la société et sur lesquels nous reviendrons. Autant il convient d'observer des rituels à ces occasions, autant il faut en observer aussi au moment de la confection même du masque. En effet, $\mathrm{du}$ fait de cette origine divine du masque, on ne saurait le fabriquer ni organiser ses sorties sans ces rituels qui rappellent les relations qu'il entretient avec les hommes de la société.

\section{Le masque est un être social}

Le masque joue un rôle prépondérant dans la vie sociale de l'homme et dans la société tout entière. Il est présent à la naissance (sociale et non biologique) de l'homme à travers les trois étapes essentielles de l'initiation ; cette initiation faite d'actes symboliques auxquels est présent le masque participe à la socialisation de l'individu, à lui donner une place et une fonction dans la société.

Outre l'initiation, le masque est un élément-clé des funérailles en pays bobo : en effet, c'est lui qui annonce le décès d'un homme âgé : il est présent à son enterrement, à ses obsèques et à ses funérailles selon un rituel précis et connu de tous.

Enfin, il est au cœur des différentes réjouissances du village, notamment les fêtes rituelles qui marquent les différents moments de la vie sociale : début des travaux champêtres, fête des récoltes, nouvel an traditionnel, etc. A chacune de ces étapes correspond son type de masque.

\section{Le masque est un objet d'art}

Malgré les dimensions sacrée et sociale du masque, il n'en demeure pas moins un objet d'art car sa confection est toujours guidée par un souci esthétique : en effet, le masque est conçu comme l'étalon de la beauté chez les Bobo (une expression ne ditelle pas « être beau comme le masque» ?).

C'est pourquoi, qu' elle que soit sa forme, un soin particulier est observé dans sa réalisation plastique, dans le choix des couleurs et surtout au moment de sa danse. Car, au-delà des circonstances qui occasionnent la présence du masque parmi les hommes, le critère fondamental de son appréciation est esthétique : est-il réalisé conformément aux normes artistiques ? Danse-t- il bien ? Est-il beau à regarder ? Autant de questions dont les réponses permettent de juger le travail de ceux qui ont été commis à la tâche.

\section{Le masque comme institution}

Nous avons déjà rappelé les principes essentiels du mythe fondateur du masque malgré les diverses versions : révélation à l'homme dans la brousse, découverte de sa 
nature par celui-ci à la condition d'observer un rituel, domptage du masque, retour de l'homme au village avec le masque. A partir de cette démarche et de l'adoption du masque par la communauté entière, il s'est imposé à celle-ci la nécessité de perpétuer ce mythe à travers les rites initiatiques et les principaux moments de la vie de l'homme. C'est ainsi que le masque se trouve au cœur de la société, rythmant sa vie au quotidien aussi bien que dans la durée. Et les initiations constituent les principales étapes de socialisation de l'individu ; elles se déroulent en trois temps :

- Entre 7 et 10 ans : on procède à la révélation du secret et du mystère du masque à l'enfant. Cette révélation comporte une charge et une dimension psychologiques importantes : il s'agit d'amener l'enfant à lutter avec le masque et à le terrasser ; après quoi il lui est révélé la vérité à savoir que c'est un homme qui s'est « déguisé ».

- Cette révélation est suivie d'une interdiction formelle d'en faire à son tour la révélation à une femme sous peine de mort. A partir de cette première initiation, l'enfant franchit la première grande étape de sa vie : la fillette commence à s'exercer aux tâches ménagères, domaine de la femme par excellence tandis que le garçonnet commence à suivre les hommes : il s'occupe de la gestion du poulailler et de l'étable et est autorisé à suivre les masques, exerçant ainsi le début de son statut officiel d'adolescent. Par rapport aux masques, il va, une dizaine d'années durant, être soumis à toutes les corvées relatives à la sortie des masques : collecte des matériaux servant à la fabrication des masques, nettoyage des aires de danse, ramassage du bois pour le feu pendant la danse nocturne des masques, etc.

- Entre 17 et 20 ans a lieu la grande initiation constituée d'épreuves physiques, psychologiques, morales et éthiques qui se déroulent en brousse, à la « source » du masque, comme pour rappeler à l'homme la suprématie de la brousse sur le village. Elle concerne exclusivement les adolescents qui auront satisfait à leur statut et qui, de ce fait, auront été jugés aptes et surtout dignes de franchir cette deuxième étape, la plus importante de la vie en rapport avec le statut qu'elle leur confère : les initiés de la même année forment une classe d'âge (la communauté est composée des différentes classes d'âge du village, avec des correspondances dans les villages environnants); cette intégration de l'individu dans la communauté lui donne des droits (autorisation de se marier, prise de parole publique, indépendances matérielles, etc.). Mais aussi des devoirs (défense de la société, respect des aînés, soumission à leur autorité, etc.).

- Deux ans après cette grande initiation, la classe d'âge franchit la troisième étape avec l'apprentissage de la langue des masques. Le masque n'étant pas un être humain, il est formellement interdit de lui parler dans la langue bobo en public : $c^{\prime}$ est pour cela que l'initié qui entre de plein pied dans le monde des adultes, des hommes, doit pouvoir communiquer avec le masque dont, du reste, il 
assure la gestion. Cet apprentissage consiste en des épreuves mnémotechniques par lesquelles l'initié apprend les caractéristiques de chaque élément vivant de la terre (animaux et végétaux) en mémorisant les paroles qui le fondent. Ces épreuves mnémotechniques s'accompagnent d'épreuves physiques d'endurance qui durent sept jours durant lesquels la classe d'âge des initiés est coupée du village et est sous la direction autoritaire de celle de ses aînés.

A travers ces trois principales étapes de la vie de l'homme liées au masque, on comprend alors l'importance que celui-ci a dans la société bobo traditionnelle. Elément de socialisation de l'individu et d'organisation de la société, le masque marque la vie individuelle et collective de la communauté. Il est le moteur, le régulateur de la vie communautaire à travers les normes et valeurs dont il est le vecteur et qui permettent à la communauté de s'identifier, de se reconnaître, de se distinguer des autres, bref il lui permet de constituer un champ autonome. L'analyse de cette autonomie qui nous permettra de mettre le masque en rapport avec la littérature comme institution passe par une comparaison entre les deux champs dont la première étape va consister à montrer l'autonomie du champ du masque ; ensuite, nous déterminerons les principales étapes de la vie de la littérature et du masque, ce qui nous permettra de conclure à l'existence de l'institution du masque.

Pour comprendre comment le masque pourrait fonctionner comme une institution, il convient tout d'abord de rappeler que l'institution est un ensemble de normes qui s'appliquent à un domaine d'activités particulier et qui définissent une légitimité s'exprimant dans une charte ou un code. A partir de ce rappel, nous allons voir comment le masque, à l'image de la littérature, fonctionne comme une institution dans la structure sociale, quelle place il occupe et comment il s'articule à d'autres ensembles.

Il y a trois niveaux d'appréciation et d'analyse de l'institution :

- Les modes d'organisation, c'est-à-dire la conservation des éléments d'un groupe, leur intégration dans le système de production et la réponse aux besoins du groupe. Ainsi chaque institution constitue un secteur spécifique d'activités et de pratiques organisé sur un mode particulier.

- la socialisation des individus qui est assurée par l'imposition de systèmes et de normes, ce qui permet la reproduction des rapports sociaux, favorise l'action éducative. Elle constitue un lieu de domination et de subordination idéologique : l'imposition des institutions se fait dans le mode de découpage des pratiques sociales et dans les conditions de possibilité et d'exercice de ces pratiques.

- la présence d'une idéologie dans l'institution qui renvoie automatiquement à un pouvoir en tant que force d'exécution au service de classes dominantes à travers un appareil répressif et un appareil idéologique. 
En partant donc de ces considérations, on peut affirmer que le masque est une institution, à la fois comme une organisation autonome, un système de socialisation et un appareil idéologique.

En tant qu'organisation autonome, la société des masques fonctionne selon des normes qui vont de la base de la société jusqu'au sommet : la société des masques exclut la gent féminine depuis la puberté jusqu'à la fin de sa fécondité. Elle organise la société en classes d'âge selon un système pyramidal. Elle régule la vie de la communauté selon un calendrier individuel et collectif.

Comme système de socialisation, le masque organise la société des hommes en classes d'âge en fonction de la date d'initiation de chaque individu, les individus initiés en même temps constituant une classe d'âge. Cette initiation donne des droits aux impétrants tout en leur imposant des devoirs dont l'observance assure la vie et la survie du groupe.

Le masque en tant qu'appareil idéologique transmet de classes d'âge en classes d'âge, de générations en générations les valeurs propres à la société. Les droits et devoirs dévolus à chaque individu dans sa classe d'âge et à chaque classe d'âge ne sauraient être piétinés pour les uns et transgressés pour les autres sans conséquences sous forme de sanctions plus ou moins importantes selon la gravité de la faute commise.

Après avoir ainsi montré le fonctionnement du masque comme un champ autonome à l'intérieur duquel les normes et les valeurs sont transmises selon des codes bien connus et acceptés de tous, nous allons maintenant comparer la littérature et le masque sous l'angle de la création artistique. Pour bien réussir cette étude comparée, nous avons procédé à un découpage de la création littéraire et de la vie du masque en six étapes correspondant chacune à un temps fort de la vie de l'œuvre d'art.

- Etape 1 : l'auteur de l'œuvre d'art. Il s'agit ici d'identifier le créateur, de voir comment il procède dans sa création. Cette étape demande une considération minutieuse du masque pour bien appréhender sa nature et surtout le mode de sa création car, appartenant à toute la collectivité, et connaissant la nature collectiviste de la société traditionnelle, la création relève ici plus de collectif que de l'individuel comme c'est le cas avec la littérature.

- Etape 2 : la réalisation de l'cuvre. Après l'identification du créateur, de l'artiste, l'étape suivante consiste à analyser comment l'œuvre d'art prend forme, comment elle est réalisée, le processus par lequel l'artiste la crée.

- Etape 3 : la production de l'œuvre. Par cette étape, il faut entendre le contrôle de la création artistique en fonction des canons et des critères selon lesquels elle est réalisée et ce qui l'autorise à être considérée véritablement comme une œuvre artistique.

- Etape 4 : la diffusion de l'œuvre. L'œuvre d'art ne saurait exister pour elle-même ; elle doit être diffusée afin d'être connue et appréciée. 
- Etape 5 : la « consommation » de l'œuvre d'art. La diffusion, ayant pour but la circulation de l'œuvre d'art, doit déboucher sur sa « consommation » au sens de son appropriation par le public auquel elle est par essence destinée.

- Etape 6 : la légitimation de l'œuvre d'art. C'est la dernière étape qui consiste à reconnaître la conformité de l'œuvre aux canons de création à travers un certain nombre de structures ; l'analyse du fonctionnement de ces structures, de même que celle des conditions de la légitimation constituent un centre d'intérêt particulier.

Après avoir ainsi présenté et expliqué ces étapes, nous allons élaborer un tableau comparatif qui nous permettra de conclure que le masque fonctionne comme l'institution de la littérature.

\section{LITTÉRATURE}

Etape 1 : L'auteur

- sa décision d'écrire est individuelle ;

- son choix du thème et du genre est libre ;

- il a aussi la possibilité d'écrire sur commande.

\section{Etape 2 : Le manuscrit}

- il s'agit de la matérialisation d'une idée librement choisie ;

- ses seules contraintes sont d'ordre moral, éthique ;

- il est soumis au respect des règles du genre.

\section{Etape 3 : L'édition}

- c'est le contrôle et la vérification de la conformité aux canons et normes du genre ;

- c'est la mise à disposition du public de l'ouvrage fini.

\section{MASQUE}

\section{Etape 1 : L'auteur}

- est anonyme, parfois collectif ;

- il agit toujours sur décision collective et sur commande ;

- il est soumis à des rituels avant de commencer son travail.

\section{Etape 2 : La confection - réalisation}

- la réalisation se fait selon des canons arrêtés en commun, imposés et permanents ;

- la liberté de l'artiste est contrôlée ;

- le travail peut être collectif avec la participation de plusieurs artistes.

\section{Etape 3 : La sacralisation rituelle}

- c'est le contrôle et la vérification de conformité aux canons et normes et rite ;

- il y a l'intégration dans le circuit de production artistique (spectacle) par le préalable du rituel : c'est la sacralisation rituelle.

\section{Etape 4 : La diffusion}

- mise à la disposition du public ;

Etape 4 : La production de spectacle

- les structures appropriées : librairies, bibliothèques ;

- disponibilité permanente de l'ouvrage sauf en cas d'épuisement de l'édition.
- mise à la disposition du public ;

- les structures appropriées sont des cadres conventionnels et rituels (décès, funérailles, réjouissance, initiations, etc.) ;

- la disponibilité de l'œuvre d'art est contrôlée par les rituels auxquels elle est soumise. 


\section{Etape 5 : La lecture}

- libre circulation des ouvrages ;

- libre consommation par les lecteurs ;

- libre appréciation : individuelle, collective et spécialisée ;

- les canons d'appréciation sont multiples et variés ;

- le destin de l'œuvre est permanent car elle est disponible à tout moment sauf en cas d'épuisement de l'édition.

\section{Etape 5 : L’appréciation artistique}

- selon la réalisation artistique du masque et sa production artistique lors de la danse, la prestation individuelle du masque est aussi appréciée en fonction des canons connus de tous ;

- l'appréciation est limitée dans le temps en fonction des circonstances de la sortie des masques.

\section{Etape 6 : La légitimation}

- structures institutionnelles : prix, académies, enseignement, critique ;

- appréciation normative postérieure à l'oeuvre ;

- critique spécialisée et individuelle.

\section{Etape 6 : La légitimation}

- plénitude du masque à travers sa prestation: acclamations du public, interpellation dans la langue du masque ;

- immédiateté, simultanéité entre la production artistique et la critique ;

- la critique est spécialisée, collective et publique ;

- les circonstances rituelles impliquent une organisation méthodique de la production ;

- les canons d'appréciation connus de tous et communs à tous les masques du même genre ;

- le destin du masque est cyclique, il y a un renouvellement permanent lié aux rites de sortie des masques;

- le masque c'est plus l'idée, le concept que l'objet qui peut être sujet à détérioration et donc renouvelable.

\section{Conclusion}

La littérature est un domaine de création artistique qui fonctionne comme une institution dont les acteurs sont clairement identifiés et les instances tout aussi connues. La sociologie de la littérature a permis de montrer le mécanisme de ce fonctionnement et s'est imposée comme une branche des études littéraires ou sociologiques.

En partant des acquis de cette approche et fort de notre expérience en sociologie de l'art concernant le masque, nous nous sommes demandé si le masque ne pouvait pas être considéré comme une institution au même titre que la littérature. Pour répondre à cette interrogation, il nous a fallu d'abord définir l'institution de la littérature ; ensuite nous avons analysé le masque en partant de la définition de sa nature et de ses fonctions dans la société traditionnelle bobo du Burkina Faso. Cela nous a conduit à 
mettre en évidence une similitude entre le fonctionnement de la littérature et celui du masque à travers une mise en miroir de la place et de la fonction des différents acteurs et structures requises pour la vie de la littérature et du masque.

$\mathrm{Au}$ terme de notre étude, notre conviction est faite que le masque, au-delà du spectacle que ses sorties procurent au public et des rites auxquels il participe, est une institution à l'instar de la littérature : les acteurs et les structures ont été clairement identifiés avec leurs différents rôles. Et comme le disait Claude Lévi-Strauss, «pas plus que les mythes, les masques ne peuvent s'interpréter en eux-mêmes et par euxmêmes comme des objets séparés ; à chaque type de masque se rattachent des mythes, qui ont pour objet d'expliquer leur origine légendaire ou surnaturel » (Lévi-Strauss $1975: 70)$.

\section{Notes}

1 Dans un article intitulé Le masque et sa fonction sociale chez les Bobo (Sanou 1995), nous avons démontré le caractère monothéiste de la société bobo dont découle l'appellation puissances spirituelles au lieu de divinités qui feraient penser à une société polythéiste.

\section{Bibliographie}

Caillois, Roger. 1978. Les Jeux et les Hommes. Le masque et le vertige. Paris: NRF, Gallimard.

Casanova, Pascale. 1999. La République mondiale des Lettres. Paris: Seuil.

Chanady, Amaryll. L'institution littéraire et l'exclusion de l'autochtone en Amérique latine. Actes du colloque Repenser la culture. <www.pum.umontreal.ca/revues/surfaces/vol2/chanad-f.html> Accédé: 30.04 .2005

Dubois, Jacques. 1978. Linstitution de la littérature. Paris; Bruxelles: Nathan : Labor.

Durand, Pascal. 2001. Introduction à la sociologie des champs symboliques. Les champs littéraires africains. Paris: Karthala

Grassin, Jean-Marie. ed. Institution. Dictionnaire International des Termes littãraires / International Dictionary of Literary Terms. <www.ditl.info> Accédé: 02.05.2005.

Le Moal, Guy. 1982. Les Bobo. Nature et fonctions des masques. Paris: ORSTOM.

Lévi-Strauss, Claude. 1975. La voie des masques. Genève: Editions de l'Art Albert Skira.

Millogo, Louis. 1988. Pour une symbiose des genres artistiques. La sortie des masques chez les Bobo: un art total. Annales de l'Université de Ouagadougou. Série A. Sciences humaines et sociales, Numéro spécial. Décembre : 75-88.

Nao, Oumarou .1996. Innovation et évolution dans l'iconographie des masques chez les Bobo méridionaux du Burkina Faso. Annales de l'Université de Ouagadougou. Série A. Sciences humaines et sociales. Vol.VIII: 71-104.

Sanou, Salaka. 1994. Le spectacle et sa fonction sociale. Cahiers $d u$ Cerleshs $N^{\circ} 11: 134-147$. 255. 1995. La fonction sociale du masque chez les Bobo de Tondogosso. Cahiers $d u$ Cerleshs $\mathrm{N}^{\circ}$ 12: 237-

2001. Le masque entre tradition et modernité chez les Bobo de Tondogosso. Annales de l'Université de Ouagadougou. Série A. Sciences humaines et sociales. Vol.XIII :197-211

. 2003. Rapport de synthèse en vue de l'Habilitation à diriger les recherches. Limoges: Université de Limoges.

Sawadogo, Raya Benjamin. 1979. Fonction et esthétique des masques mossis de la Haute-Volta. Thèse de doctorat $3^{\text {ème }}$ cycle. Université Paris I. 